\title{
Conhecimento da equipe de enfermagem frente ao paciente transgênero
}

\author{
Knowledge of the nursing team regarding transgender patients \\ Conocimiento del equipo de enfermería sobre pacientes transgénero
}

Júlia Cristine Gomes de Lima

ORCID: https://orcid.org/ 0000-0001-9073-5163 Centro Universitário Estácio de Sergipe, Brasil

E-mail: cristinejulia@outlook.com

Neizyane Santos Oliveira

ORCID: https://orcid.org/0000-0002-5793-5347 Centro Universitário Estácio de Sergipe, Brasil

E-mail: neizyane-bol@ hotmail.com

André Luiz de Jesus Morais

ORCID: https://orcid.org/0000-0003-4889-8297 Centro Universitário Estácio de Sergipe, Brasil

E-mail: enfermeiro.andre@ hotmail.com

Weber de Santana Teles

ORCID: https://orcid.org/0000-0003-1770-8278

Centro de Hemoterapia de Sergipe, Brasil

E-mail: arteecura@hotmail.com

Max Cruz da Silva

ORCID: https://orcid.org/0000-0002-6944-5986

Faculdade Pio Décimo, Brasil

E-mail: maxlfi@hotmail.com

Ruth Cristini Torres

ORCID: https://orcid.org/0000-0002-8664-192X Instituto de Hematologia e Hemoterapia de Sergipe, Brasil

E-mail: ruthcristini@gmail.com

Ângela Maria Melo Sá Barros

ORCID: https://orcid.org/0000-0003-4087-3247

Universidade Federal do Rio de Janeiro, Brasil

E-mail: angelsamelo@ @otmail.com

Marcel Vinícius Cunha Azevedo

ORCID: https://orcid.org/0000-0002-5312-3333

Centro Universitário Estácio Sergipe, Brasil

E-mail: marcelvinicius49@gmail.com

Paulo Celso Curvelo Santos Junior

ORCID: https://orcid.org/0000-0001-5834-6782

Universidade Tiradentes, Brasil

E-mail: paulo.curvelo.jr@gmail.com

Alejandra Debbo

ORCID: https://orcid.org/0000-0002-7743-5921

Universidade Federal de Sergipe, Brasil E-mail: aledebbo@hotmail.com

Taíssa Alice Soleade Calasans

ORCID: https://orcid.org/0000-0003-0460-4437

Universidade Tiradentes, Brasil

E-mail: taissa.asc@gmail.com

Maria Hozana Santos Silva

ORCID: https://orcid.org/0000-0001-5742-5366

Faculdade Ages de Medicina, Brasil

E-mail: hosana_p@hotmail.com

\section{Resumo}

Objetivo: Analisar a produção científica nacional e internacional sobre nível de conhecimento da equipoe de enfermagem frente ao paciente trans. Método: Revisão integrativa da literatura nas bases de dados Biblioteca Virtual em Saúde (BVS) e Public Medline (PubMed), utilizando-se os cruzamentos dos descritores: Assistência de enfermagem, Minorias sexuais e de gênero, Pessoas transgênero, e seus correspondentes na língua inglesa Nursing Care, Sexual and Gender Minorities e Trangender persons. Em ambos os idiomas esses termos foram combinados utilizando-se o operador booleano and. Elaborou-se um instrumento para a coleta das informações, composto pelos seguintes itens: título, revista, autores/ano de publicação e conclusão. Resultados: Foram incluídos 11 estudos, 
publicados entre 2016-2020, posteriormente dividos em duas categorias. Considerações finais: é evidente a necessidade dos desafios a serem vencidos no que tange a atenção a saúde da comunidade transgênero.

Palavras-chave: Assistência de enfermagem; Minorias sexuais e de gênero; Pessoas transgênero.

\begin{abstract}
Objective: To analyze the national and international scientific production on the level of knowledge of the nursing team regarding trans patients. Method: Integrative literature review in the Virtual Health Library (BVS) and Public Medline (PubMed) databases, using the intersections of the descriptors: Nursing care, Sexual and gender minorities, Transgender people, and their language counterparts English Nursing Care, Sexual and Gender Minorities and Trangender persons. In both languages these terms were combined using the Boolean operator and. An instrument was developed to collect information, consisting of the following items: title, journal, authors/year of publication and conclusion. Results: 11 studies were included, published between 2016-2020, later divided into two categories. Final considerations: it is evident the need for challenges to be overcome regarding the health care of the transgender community.
\end{abstract}

Keywords: Nursing care; Sexual and gender minorities; Transgender people.

\title{
Resumen
}

Objetivo: Analizar la producción científica nacional e internacional sobre el nivel de conocimiento del equipo de enfermería sobre los pacientes trans. Método: revisión integradora de la literatura en las bases de datos de la Biblioteca Virtual en Salud (BVS) y Public Medline (PubMed), utilizando las intersecciones de los descriptores: Atención de enfermería, Minorías sexuales y de género, Personas transgénero y sus contrapartes lingüísticas Inglés Atención de enfermería, Sexual y género Minorías y personas transgénero. En ambos idiomas, estos términos se combinaron utilizando el operador booleano y. Se desarrolló un instrumento para la recolección de información, compuesto por los siguientes ítems: título, revista, autores / año de publicación y conclusión. Resultados: Se incluyeron 11 estudios, publicados entre 2016-2020, luego divididos en dos categorías. Consideraciones finales: es evidente la necesidad de superar desafíos en la atención de la salud de la comunidad transgénero.

Palabras clave: Atención de enfermería; Minorías sexuales y de género; Personas transgénero.

\section{Introdução}

Pessoas transgêneros são aquelas em que o sexo biológico atribuído ao nascimento não condiz com a identidade de gênero. São indivíduos que vivem fora dos padrões de gênero e sexualidade impostas pela sociedade, estando vulneráveis a terem seus direitos básicos ameaçados e, portanto, garantir saúde de forma equânime é um desafio a ser vencido (Olvieira, Silva, Souza, 2019 \& Al, et al., 2017).

A história da homossexualidade no maior país da América do Sul é marcada pelo prejulgamento, devido os atavismos culturais, agressão homofóbica, em relação a personalidade e atuação dos LGBTs na sociedade (Costa, et al., 2015).

A repulsão, repugnância e preconceito que alguns indivíduos alimentam contra os homossexuais, lésbicas, bissexuais e transexuais é considerado como homofobia, entretanto, Junqueira (2007), estabelece que esse elóquio doentio são definidos por outros estudiosos como um agente que restringe a condição de cidadão como proibitório aos direitos preconizados pelo Sistema Único de Saúde.

Pesquisadores conceituam a homofobia, como um termo comum utilizado pela população para manifestar posicionamentos, hábitos, pejorativos de fúria, malevolência e repulsão contra indivíduos que são gays e lésbicas, transexuais e as travestis (Fazzano, et al., 2015). No entanto para alguns pesquisadores informa que homofobia é uma maneira de manifestar uma opinião e que não se enquadra como uma ação criminosa em si, mas a sua manifestação nefasta é considerada crime, mas as sensibilizações a prática da homofobia devem de enquadrar nos instrumentos de punição policial. (Rigaud, 2014)

A paridade, liberdade e a honra do ser humano é consagrado pela Constituição Federal, tendo em vista que qualquer tipo de discriminação deve ser impedido. A inexistência de regulamentos direcionados a comunidade LGBTs, não traduz que essas pessoas poder ficar à mercê dos preconceitos e agressões dos que permanecem na ignorância (Jota, 2017). 
As agressividades e insultos aos portadores da sexualidade trans tem acontecido no decorrer de vários anos e em diversas localidades, tendo em vista os arquétipos de intolerância, aversão e exclusão por parte de um grupo descriminado, sem proteção por parte dos órgãos governamentais, que não oferece políticas públicas e de saúde e de segurança a este seguimento social (Borges, et al., 2017).

A Política Nacional de Saúde Integral da População LGBTQ+ surgiu com o intuito de favorecer equidade no SUS, de modo que a população possua mais acesso à saúde integral e atenção especial às demandas especificas, além de eliminar preconceito, discriminação, violência, entre outros. Alguns programas nacionais e mundiais preconizam discussão e qualificação dos direitos e saúde da população Lésbicas, Gays, Bissexuais, Transexuais, Queer, Intersexo, Assexuais e Pansexuais (LGBTQIAP+), porém esse assunto é marginalizado das grandes discussões, sejam elas nas escolas médicas ou na atenção básica (Brasil, 2013, Albuquerque, Botelho \& Rodrigues, 2019).

Nesse contexto, existem deficiências dos profissionais ao atender um paciente trans e prestar os devidos cuidados relacionado às suas necessidades de saúde. Assim, os profissionais têm dúvidas quanto à conduta a ser realizada e como chamar esse paciente na abordagem, ficam receosos em discutir sobre sua sexualidade por medo de causar constrangimento ou insultá-los. Acredita-se que esses desafios estão relacionados ao raciocínio heteronormativo, e a uma sociedade que impõe a cisgeneridade (Moscheta, Souza \& Santos, 2016).

A justificativa para esse estudo se deu através de questionamentos dos pesquisadores a respeito da atual situação da abordagem da equipe de enfermagem aos pacientes transgêneros, visto que na academia não há disciplina específica voltada para esse público, onde percebemos a necessidade de buscar conhecimento sobre essa realidade. Assim, o presente estudo tem como objetivo buscar a produção científica nacional e internacional sobre o as principais dificuldades da equipe de enfermagem frente ao paciente trans., seja dentro da rede hospitalar de saúde ou nas unidades básicas ou de pronto atendimento, contribuindo assim para uma melhor atuação da equipe de enfermagem em todos os setores de sua permanência.

\section{Metodologia}

Trata-se de uma revisão integrativa de literatura: um método que tem como finalidade sintetizar resultados obtidos em pesquisas sobre um tema ou questão, de maneira sistemática, ordenada e abrangente. Possuem métodos criteriosos para fornecer conhecimentos acerca de um problema de pesquisa serem posteriormente avaliados por um profissional habilitado clinicamente. Foi elaborado por meio de seis etapas: 1. Identificação do tema e seleção da hipótese; 2. Estabelecimento de critérios para inclusão e exclusão de estudos; 3. Categorização dos estudos; 4. Avaliação crítica dos estudos; 5. Interpretação dos resultados; 6. Apresentação da revisão (Mendes, Silveira \& Galvão, 2008).

Desta maneira, para guiar o estudo elaborou-se indagações a equipe de enfermagem apresenta dificuldade no atendimento a um paciente trans? A equipe de enfermagem compreende questões de transgeneridade dentro de seu serviço de atuação profissional bem como os direitos dessa população?

Este estudo teve como objetivo entender sobre as dificuldades da equipe de enfermagem frente ao paciente trans, para responder a questão do estudo: quais as principais dificuldades da equipe de enfermagem que é composta por enfermeiros, técnicos e auxiliares de enfermagem, dentro de seu ambiente de trabalho, no atendimento às pessoas trans? Para isso foi utilizado a estratégia pacientes; intervenção; controle; desfecho que é utilizada para auxiliar o que de fato a pergunta da pesquisa deve especificar. Realizado no período de março de 2020 a novembro de 2020, foram incluídos na pesquisa artigos originais, realizadas em humanos, dos últimos 05 anos, com idiomas português e inglês, e que abordassem o tema em estudo; foram excluídos os artigos em duplicidade e aqueles que não tratassem diretamente da temática proposta. $\mathrm{O}$ recorte temporal 
foi escolhido com um ano de posse do atual governo por termos observado uma baixa significativa de investimentos públicos no quesito particular da população trans aqui intencionalmente estudada.

Após a leitura dos títulos e resumos dos artigos que havia concordância com o objetivo do trabalho foram selecionados os que foram lidos na íntegra. Foi realizada a busca e organização das temáticas, por meio da análise das publicações indexadas nas seguintes bases de dados: Biblioteca Virtual em Saúde (BVS) e Public Medline (PubMed), utilizando-se os cruzamentos dos descritores: Assistência de enfermagem, Minorias sexuais e de gênero, Pessoas transgênero, provenientes do Descritores em Ciências da Saúde (DeCS). E seus correspondentes na língua inglesa: Nursing Care, Sexual and Gender Minorities e Trangender persons. Em ambos os idiomas esses termos foram combinados utilizando-se o operador booleano and.

Na pesquisa foram encontrados 286 estudos: 09 publicações na BVS e 277 na Pubmed. Após a seleção dos filtros e análise dos estudos, mediante leitura de títulos e resumos, obteve-se um total de 49 estudos, os quais foram lidos na íntegra. Após leitura completa foram selecionados os 11 artigos que atingiram o objetivo e responderam ao problema de pesquisa proposto, como demonstra a Figura 1.

Figura 1: Fluxograma na modalidade Prisma da seleção de publicações.

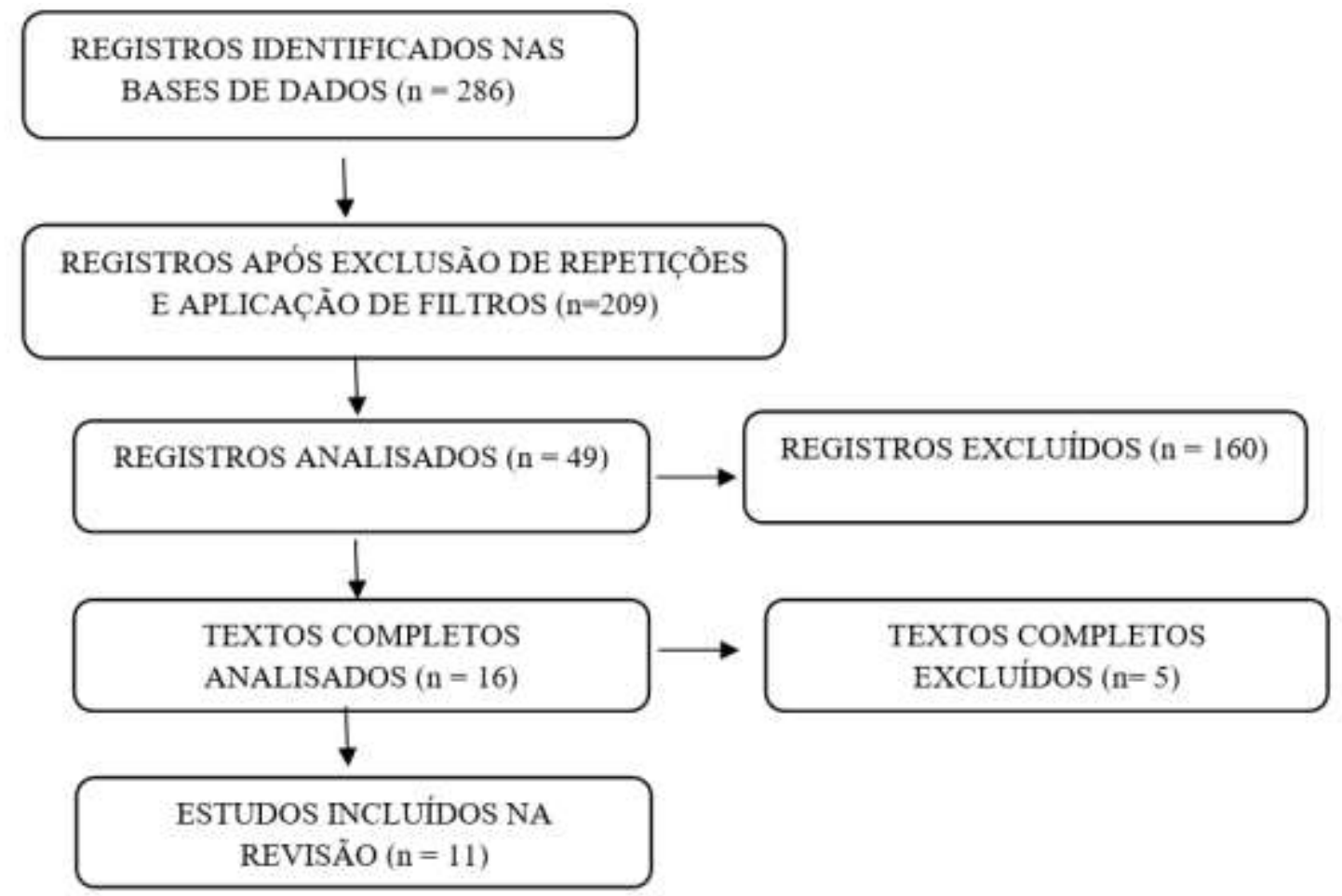

Fonte: Elaboração própria.

Elaborou-se um instrumento para a coleta das informações, composto pelos seguintes itens: título, revista, autores/ano de publicação e conclusão. Os artigos analisados foram organizados numa planilha do software Microsoft Office Excel $2013^{\circledR}$ e expressos em quadro, que constituiu o banco de dados do estudo. Os artigos foram agrupados por similaridade e temática pertinente, originando categorias que foram apresentadas, analisadas e discutidas mediante a literatura disponível relacionada ao tema. 
Por se tratar de uma pesquisa bibliográfica, os dados obtidos por meio dessa, seguiram princípios éticos previstos na lei dos direitos autorais $12.853 / 13$, que dispõe sobre a gestão coletiva de direitos autorais.

\section{Resultados}

Dos 11 artigos selecionados, 02 foram de 2019, 02 de 2018, 04 de 2017 e 03 de 2016. A partir de então foi possível analisar os artigos integrando as ideias dos autores, possibilitando uma análise coerente com o objetivo deste estudo.

Quadro 1: Distribuição dos estudos incluídos na revisão integrativa. Aracaju-SE, 2020.

\begin{tabular}{|c|c|c|c|}
\hline TÍTULO & REVISTA & $\begin{array}{l}\text { AUTOR/ANO DE } \\
\text { PUBLICAÇÃO }\end{array}$ & CONCLUSÃO \\
\hline $\begin{array}{l}\text { Atenção integral à saúde da } \\
\text { população LGBT: Experiência de } \\
\text { educação em saúde com agentes } \\
\text { comunitários na atenção básica }\end{array}$ & $\begin{array}{l}\text { Revista Brasileira } \\
\text { de Medicina de } \\
\text { Família e } \\
\text { Comunidade }\end{array}$ & $\begin{array}{l}\text { Albuquerque, Botelho } \\
\text { \& Rodrigues, } 2019 .\end{array}$ & $\begin{array}{l}\text { Os profissionais médicos e enfermeiros acabam } \\
\text { se sentindo desconfortáveis e despreparados } \\
\text { para abordarem tal tema, corroborando em } \\
\text { marginalização da população LGBT. }\end{array}$ \\
\hline $\begin{array}{l}\text { A Política Nacional de Saúde } \\
\text { Integral de Lésbicas, Gays, } \\
\text { Bissexuais e Transgêneros (LGBT) } \\
\text { e o acesso ao Processo } \\
\text { Transexualizador no Sistema Único } \\
\text { de Saúde (SUS): avanços e desafios }\end{array}$ & $\begin{array}{l}\text { Ciência \& Saúde } \\
\text { Coletiva }\end{array}$ & $\begin{array}{l}\text { Popadiuk, Oliveira \& } \\
\text { Signorelli, } 2017 .\end{array}$ & $\begin{array}{l}\text { A garantia efetiva de saúde ainda não é } \\
\text { realidade das pessoas trans, visto que garantir } \\
\text { saúde não é somente proporcionar práticas } \\
\text { voltadas a condição patológica, mas também ao } \\
\text { encontro da concepção ampliada de saúde. }\end{array}$ \\
\hline $\begin{array}{l}\text { O direito à saúde de pessoas trans* } \\
\text { no Distrito Federal: entre o direito } \\
\text { de existir e o direito à equidade }\end{array}$ & $\begin{array}{l}\text { Cadernos Ibero- } \\
\text { americanos de } \\
\text { Direito Sanitário }\end{array}$ & $\begin{array}{l}\text { Oliveira, Silva \& Souza, } \\
2019 .\end{array}$ & $\begin{array}{l}\text { Pessoas que vivem fora dos padrões de gênero e } \\
\text { sexualidade impostas pela sociedade não são } \\
\text { reconhecidas como seres humanos, portanto } \\
\text { garantir saúde de forma equânime é um desafio } \\
\text { a ser vencido. }\end{array}$ \\
\hline Disforia de Gênero & $\begin{array}{l}\text { Sociedade } \\
\text { Brasileira de } \\
\text { Pediatria }\end{array}$ & Vila, et al., 2017. & $\begin{array}{l}\text { Os profissionais reagem negativamente no } \\
\text { atendimento a esse público, favorecendo a uma } \\
\text { menor procura destes ao serviço de saúde. }\end{array}$ \\
\hline $\begin{array}{l}\text { Assistência à saúde da população } \\
\text { LGBT em uma capital brasileira: o } \\
\text { que dizem os Agentes Comunitários } \\
\text { Saúde? }\end{array}$ & $\begin{array}{l}\text { Tempus actas de } \\
\text { saúde coletiva }\end{array}$ & $\begin{array}{l}\text { Guimarães, R. C. P. Et } \\
\text { Al., } 2017\end{array}$ & $\begin{array}{l}\text { É importante investir dentro das instituições de } \\
\text { saúde metodologias ativas que proponham aos } \\
\text { profissionais expor e trabalhar valores e crenças } \\
\text { e reconhecer possíveis preconceitos e estigmas, } \\
\text { para que seja possível desconstruí-los, o que } \\
\text { contribuirá para sua formação ética e técnica }\end{array}$ \\
\hline $\begin{array}{l}\text { Serviços de saúde para lésbicas, } \\
\text { gays, bissexuais e } \\
\text { travestis/transexuais }\end{array}$ & $\begin{array}{l}\text { Revista de } \\
\text { enfermagem } \\
\text { UFPE online }\end{array}$ & Oliveira, et al., 2018. & $\begin{array}{l}\text { Os profissionais de saúde reconhecem a } \\
\text { necessidade de existir equidade relacionado a } \\
\text { esse público, principalmente por conta do } \\
\text { comportamento sexual de risco, porém devido } \\
\text { aos valores sociais e culturais repulsivos, não é } \\
\text { uma realidade vivenciada }\end{array}$ \\
\hline $\begin{array}{l}\text { Communicating With Patients } \\
\text { Who Have Nonbinary Gender } \\
\text { Identities }\end{array}$ & $\begin{array}{l}\text { Annals of } \\
\text { family medicine }\end{array}$ & $\begin{array}{l}\text { Goldhammer,Malina } \\
\& \text { Keuroghlian, } \\
2018 .\end{array}$ & $\begin{array}{l}\text { Tendo em vista a diversidade de gênero } \\
\text { existente é necessário que os médicos } \\
\text { apoiem a autodefinição dos clientes sobre } \\
\text { sua identidade de gênero. A equipe de } \\
\text { saúde deve então evitar suposições, } \\
\text { perguntar qual o nome e pronome que } \\
\text { gostariam de ser tratados. }\end{array}$ \\
\hline
\end{tabular}


Continuação do Quando 1

\begin{tabular}{|c|c|c|c|}
\hline TÍTULO & REVISTA & $\begin{array}{l}\text { AUTOR/ANO DE } \\
\text { PUBLICAÇÃO }\end{array}$ & CONCLUSÃO \\
\hline $\begin{array}{l}\text { Competent persons who can treat } \\
\text { you with competence, as simple as } \\
\text { that" - An interview study with } \\
\text { transgender people on their } \\
\text { experiences of meeting health care } \\
\text { professionals }\end{array}$ & $\begin{array}{l}\text { Journal of Clinical } \\
\text { Nursing }\end{array}$ & Lindroth, 2016. & $\begin{array}{l}\text { O conhecimento em relação a população trans } \\
\text { precisa ser aumentada tanto nos ambientes } \\
\text { especializados em transgêneros quanto em } \\
\text { outros ambientes de assistência médica, a fim } \\
\text { de evitar constrangimento nas pessoas trans. }\end{array}$ \\
\hline $\begin{array}{l}\text { Do student nurses feel a lack of } \\
\text { comfort in providing support for } \\
\text { Lesbian, Gay, Bisexual or } \\
\text { Questioning adolescents: what } \\
\text { factors influence their comfort level }\end{array}$ & $\begin{array}{l}\text { Journal of } \\
\text { Advanced } \\
\text { Nursing }\end{array}$ & $\begin{array}{l}\text { Richardson, Ondracek } \\
\text { \& Anderson, } 2017 .\end{array}$ & $\begin{array}{l}\text { Os estudantes de enfermagem precisam ser } \\
\text { melhor capacitados dentro da instituição de } \\
\text { ensino, dessa forma é importante existir uma } \\
\text { disciplina que a aborde questões LGBTQIAP+. }\end{array}$ \\
\hline $\begin{array}{l}\text { Health care provision in Brazil: A } \\
\text { dialogue between health } \\
\text { professionals and lesbian, gay, } \\
\text { bisexual and transgender service } \\
\text { users }\end{array}$ & $\begin{array}{l}\text { Journal of Health } \\
\text { Psychology }\end{array}$ & $\begin{array}{l}\text { Moscheta, Souza \& } \\
\text { Santos, } 2016 .\end{array}$ & $\begin{array}{l}\text { Existe deficiência dos profissionais ao atender } \\
\text { um paciente trans e prestar os devidos cuidados } \\
\text { relacionado às suas necessidades de saúde. }\end{array}$ \\
\hline $\begin{array}{l}\text { Discrimination and Delayed Health } \\
\text { Care Among Transgender Women } \\
\text { and Men }\end{array}$ & Medical Care & $\begin{array}{l}\text { Jaffee, Shires \& } \\
\text { Stroumsa, } 2016 .\end{array}$ & $\begin{array}{l}\text { Pessoas trans com um nível de instrução mais } \\
\text { avançado acabam deixando os profissionais de } \\
\text { saúde desconfortáveis, por fazer perguntas mais } \\
\text { especificas e estes não saberem responder. }\end{array}$ \\
\hline
\end{tabular}

Fonte: Elaboração própria.

\section{Discussão}

Com a leitura e organização das informações dos estudos selecionados, foi possível categorizá-los em duas temáticas: I - Políticas públicas; II - Fragilidades da equipe de saúde no atendimento ao paciente trans.

\section{Políticas públicas}

A partir da implantação do SUS no ano de 1988 percebeu-se avanços com relação à saúde da população LGBTQIAP+. Duas grandes conquistas tidas com esforço foram o processo transexualizador e o Programa Nacional de Saúde Integral de Lésbicas, Gays, Bissexuais e Transexuais. No entanto, apenas em 2008 foram criados ambulatórios e hospitais habilitados para o processo transexualizador de modo a atender as especificidades da população trans. Porém, a garantia efetiva de saúde ainda não é realidade das pessoas trans, visto que promover saúde é necessário visualizar o indivíduo como ser biopsicossocial (Popadiuk, Oliveira \& Signorelli, 2017).

Mesmo diante das medidas criadas pelo Estado para combater discriminação e preconceito como legalização da união homoafetiva, obrigatoriedade do uso do nome social para transexuais e travestis no serviço de saúde e regulamentação do processo transexualizador pelo SUS, ainda há muita resistência da aceitação pela população geral, principalmente por ser composta de uma construção sócio-histórica cristã, patriarcal e sexista. Nesse sentido, existe uma resistência por parte da população LGBTQIAP+ em procurar o serviço de saúde, isso pode indicar discriminação evidenciado pelo padrão heteronormatividade, falta de qualificação e preconceito da equipe de saúde. (Guimarães, et al., 2017).

Considerando os direitos mais básicos da sociedade, o direito de existir é a principal discussão presente entre a população trans. O ser homem ou mulher normal definido pela sociedade heteronormativa consequentemente provoca uma 
marginalização desse grupo, fazendo com que eles se vejam cotidianamente em condições de preconceito, violência e negligência social (Oliveira, Silva \& Souza, 2019).

Com base nisso é notável o avanço da assistência específica para a população trans, entretanto, a existência desses programas não é suficiente para abranger esse grupo, pois na atenção básica não é uma temática abordada. Apesar da Unidade Básica de Saúde (UBS) funcionar como coordenação para os serviços de saúde, sendo essenciais para a divulgação e encaminhamento. Todavia, a falta de conhecimentos dos profissionais afeta diretamente o projeto terapêutico singular, a integralidade e continuidade da assistência além de desrespeitar os princípios de equidade, contribuindo para segregação desses pacientes.

Ademais, até o ano de 2017 não existia uma lei que regulamentava a retificação de prenome, sexo e imagem nos documentos pessoais, isso gerava uma dificuldade para àquelas pessoas que precisam ser reconhecidos na sociedade com sua real identidade de gênero, ficando a mercê dos laudos médicos e interpretações e exigências de cada juiz. Além disso os juízes eram mais propensos a conceder parecer favorável aqueles que já haviam realizado a cirurgia de resignação de sexo, deixando de fora a parcela de sujeitos que possuíam disforia de gênero, mas que não optava pela cirurgia (Val, et al., 2017).

\section{Fragilidades da equipe de saúde no atendimento ao paciente trans}

Levando em consideração que a UBS é porta de entrada para o atendimento da comunidade há a necessidade de estar capacitada para atender e abordar pacientes LGBTQIAP+ de modo a diminuir os índices de discriminação existente dentro desses ambientes. A discussão sobre a discriminação da população LGBTQIAP+ é pouco observada nos setores de saúde e a UBS é um ambiente propício para promover essas ações. Todavia, por ser um tema não abordado na academia os profissionais acabam se sentindo desconfortáveis e despreparados para abordarem tal tema, corroborando a marginalização da população (Albuquerque, Botelho \& Rodrigues, 2019).

Nessa perspectiva, apesar dos profissionais de enfermagem considerarem pessoas LGBTQIAP+ detentoras dos direitos a saúde, também admitem que não são ofertados serviços específicos para essa população. Contudo, isso acontece em consequência aos valores morais socioculturais e religiosos repulsivos inerentes nesses profissionais, que acabam ferindo o princípio de equidade. Mesmo com a educação permanente, através de temáticas ligadas à sexualidade, continua-se com a concepção que respeitar o nome social e a possibilidade de usar um banheiro compatível com o gênero das pessoas transexuais são suficientes para garantir um sistema justo e humanizado com essa população (Oliveira, 2018 \& Duarte, 2020).

Além disso, a função do profissional de saúde é promover saúde e garantir a proteção e a ciência direitos sexuais das pessoas trans, evitando suposições gênero e/ou que compartilham experiências semelhantes (Perucchi, et al., 2018).

Dessa forma, é indispensável conhecer a pessoa em sua integralidade para então descobrir suas necessidades de saúde, viabilizando estratégias de prevenção, promoção e assistência à saúde para estimular e proporcionar uma melhor assistência (Lindroth, 2016, Albuquerque, Botelho \& Rodrigues, 2019).

Em uma sociedade na qual impõe-se que o sexo biológico seja determinante para a identidade de gênero é de suma importância não somente estratégias de educação continuada, mas também fortalecer princípios de bioética. Além disso, fomentar o comprometimento em não permitir que valores morais e/ou religiosos interfiram na assistência prestada para que, consequentemente todos os usuários podem desfrutar do princípio de equidade e favorecer o acolhimento como fundamental para um cuidado de alta qualidade (Taquette, et al., 2015)

Pessoas trans com um nível de instrução mais avançado acabam deixando os profissionais de saúde desconfortáveis, por fazer perguntas mais especificas e estes não saberem responder. Essa falta de conhecimento pode levar ao profissional agir 
de forma discriminatória, fazendo com que o paciente em questão não retorne para realizar os cuidados necessários à sua saúde (Jaffee, Shires \& Stroumsa, 2016).

Assim, o conhecimento em relação a população trans precisa ser aumentado tanto nos ambientes especializados em transgêneros quanto em outros ambientes de assistência médica a fim de evitar constrangimento nas pessoas trans e na assistência de cuidados inadequados. Portanto, o enfermeiro tem papel fundamental na promoção e luta pela equidade e justiça nos cuidados à saúde (Lindroth, 2016).

Tendo em vista a diversidade de gênero existente é necessário que a equipe de saúde apoie a autodefinição dos clientes sobre sua identidade de gênero. Devendo, assim, evitar suposições, perguntar qual o nome e pronome que gostariam de ser tratados e preencher seu formulário com termos que contemplem a identificação de gênero (Goldhammer, Malina \& Keuroghlian, 2018).

Por isso a importância de investir dentro das instituições de saúde metodologias ativas que proponham aos profissionais expor e trabalhar valores e crenças e reconhecer possíveis preconceitos e estigmas, para que seja possível (des) construí-los, o que contribuirá para sua formação ética e técnica (Guimarães, et al., 2017).

\section{Considerações Finais}

Apesar da nítida evolução na criação de sistemas específicos de assistência a pessoas trans, como ambulatório trans e projeto transexualizador, ainda há uma grande dificuldade dos profissionais de saúde no atendimento a essa população. Muitas vezes o desconhecimento e a falta de vínculo com essa comunidade são transformados em preconceito e discriminação, fazendo com que esses usuários se distanciem dos serviços de saúde, afetando a procura, adesão e continuidade do tratamento.

Tendo em vista que a enfermagem está à frente do cuidado em saúde, torna-se urgente mais capacitações para essa classe e assim, prestando uma assistência mais digna e humanizada. Além disso, os estudantes de enfermagem precisam ser melhores capacitados dentro da instituição, abordando mais questões referentes ao cuidado integral a pessoas LGBTQIAP+. Os alunos hoje serão posteriormente enfermeiros e mentores e, assim, ter em sua grade disciplinas que favoreçam esse conhecimento lhe proporciona segurança na prática em saúde voltada para esse público.

\section{Referências}

Albuquerque, M. R. T. C., Botelho, N. M. \& Rodrigues, C. C. P. (2019). Atenção integral à saúde da população LGBT: Experiência de educação em saúde com agentes comunitários na atenção básica. Rev Bras Med Fam Comunidade. 14(41), 1758.

Borges, T. Adriana, F. Noábia, M.F. \& Dantas, M.O. (2017). Políticas de saúde para lésbicas, gays, bissexuais, travestis e transexuais no Brasil: Em busca de universalidade, integralidade e equidade. II Seminário Nacional de Serviço Social e Políticas Sociais. Universidade Federal de santa Catarina.

Brasil. Ministério da saúde. (2013). Secretaria de gestão estratégica e participativa. Departamento de apoio à gestão participativa. Política nacional de saúde integral de lésbicas, gays, bissexuais, travestis e transexuais. 1(1), 32.

Costa, A. B. \& Nardi, H. C. (2015). Homofobia e Preconceito contra Diversidade Sexual: Debate Conceitual. Temas em Psicologia. 23(3), 715-726.

Duarte, D.D, et al. (2020). A pespectiva do enfermeiro no cuidado diante da pessoa trans. Research, Society and Development. 9(4), e61942845.

Fazzano, L. H. \& Gallo, A. E. (2015). Uma análise da homofobia sob a perspectivada análise do comportamento. Temas psicol. 23(3), 535- 545.

Goldhammer, H. Malina, S. \& Keuroghlian, A. S. (2018). Communicating With Patients Who Have Nonbinary Gender Identities. Annals of family medicine. $16(6), 559-562$

Guimarães, R. C. P. et al. (2017). Assistência à saúde da população LGBT em uma capital brasileira: o que dizem os Agentes Comunitários de Saúde? Tempus actas de saúde coletiva. 11(1), 121-139.

Jaffee, K. D., Shires, D.A. \& Stroumsa, D. (2016). Discrimination and Delayed Health Care Among Transgender Women and Men. Medical Care, 54(11), 1010-1016.

Junqueira, R. (2007). Homofobia: limites e possibilidades de um conceito em meio a disputas. Bagoas: estudos gays - gêneros e sexualidades. 1(1), 145-65. 
Research, Society and Development, v. 10, n. 10, e01101018394, 2021

(CC BY 4.0) | ISSN 2525-3409 | DOI: http://dx.doi.org/10.33448/rsd-v10i10.18394

Lindroth, M. (2016). Competent persons who can treat you with competence, as simple as that" - An interview study with transgender people on their experiences of meeting health care professionals, Journal of Clinical Nursing, 25(23-24), 3511-3521.

Mendes, K. D. S., Silveira, R. C. C. P. \& Galvão, C. M. (2008). Revisão integrativa: método de pesquisa para a incorporação de evidências na saúde e na enfermagem. Texto contexto - enfermagem. 17(4).

Moscheta, M. S., Souza, L. V. \& Santos, M. A. (2016). Health care provision in Brazil: A dialogue between health professionals and lesbian, gay, bisexual and transgender service users. Journal of Health Psychology. 21(3), 369-378.

Oliveira, B, P., Silva, M. A .S. \& Souza, M. S. (2019). O direito à saúde de pessoas trans* no Distrito Federal: entre o direito de existir e o direito à equidade. Cad. Ibero-amer. Dir. Sanit. 8(1), 1-163.

Oliveira, G.S., et al. (2018). Serviços de saúde para lésbicas, gays, bissexuais e travestis/transexuais. Revista de enfermagem UFPE online. 12(10), 2598-609.

Perucchi, J., Brandao, B. C. \& Vieira, H. I. S. Aspectos psicossociais da homofobia intrafamiliar e saúde de jovens lésbicas e gays. Estud. psicol. (Natal). 19(1). 67-76

Popadiuk, G. S., Oliveira, D. C. \& Signorelli, M. C. (2017). A Política Nacional de Saúde Integral de Lésbicas, Gays, Bissexuais e Transgêneros (LGBT) e o acesso ao Processo Transexualizador no Sistema Único de Saúde (SUS): avanços e desafios. Ciência \& Saúde Coletiva. 22(5), 1509-1520.

Rigaud, R. (2014). Pelo Direito de Ser. O carnaval democrático: os “mistérios e emoções” do sistema eleitoral brasileiro. 18(2).

Richardson, B. P., Ondracek, A. \& Anderson D. (2017). Do student nurses feel a lack of comfort in providing support for Lesbian, Gay, Bisexual or Questioning adolescents: what factors influence their comfort level?, Journal of Advanced Nursing, 73(5), 1196-1207.

Val, A. C. et al. (2017). Disforia de gênero. Sociedade Brasileira de Pediatria. 4(1). 